\title{
Adaptive Learning Path Recommendation based on Graph Theory and an Improved I mmune Algorithm
}

\author{
Cun-Ling BIAN ${ }^{1}$, De-Liang WANG ${ }^{1}$, Shi-Yu LIU ${ }^{1}$, Wei-Gang LU $^{1,2}$, and Jun-Yu DONG ${ }^{2}$ \\ ${ }^{1}$ Department of Educational Technology, Ocean University of China \\ Qingdao, Shandong-China \\ [e-mail: luweigang@ouc.edu.cn] \\ ${ }^{2}$ Department of Computer Science and Technology, Ocean University of China \\ Qingdao, Shandong-China \\ *Corresponding author: Wei-Gang LU
}

Received July 15, 2018; revised September 13, 2018; accepted November 23, 2018; published May 31, 2019

\begin{abstract}
Adaptive learning in e-learning has garnered researchers' interest. In it, learning resources could be recommended automatically to achieve a personalized learning experience. There are various ways to realize it. One of the realistic ways is adaptive learning path recommendation, in which learning resources are provided according to learners' requirements. This paper summarizes existing works and proposes an innovative approach. Firstly, a learner-centred concept map is created using graph theory based on the features of the learners and concepts. Then, the approach generates a linear concept sequence from the concept map using the proposed traversal algorithm. Finally, Learning Objects (LOs), which are the smallest concrete units that make up a learning path, are organized based on the concept sequences. In order to realize this step, we model it as a multi-objective combinatorial optimization problem, and an improved immune algorithm (IIA) is proposed to solve it. In the experimental stage, a series of simulated experiments are conducted on nine datasets with different levels of complexity. The results show that the proposed algorithm increases the computational efficiency and effectiveness. Moreover, an empirical study is carried out to validate the proposed approach from a pedagogical view. Compared with a self-selection based approach and the other evolutionary algorithm based approaches, the proposed approach produces better outcomes in terms of learners' homework, final exam grades and satisfaction.
\end{abstract}

Keywords: Adaptive learning, graph theory, improved immune algorithm, learning path recommendation

This work has been supported by the MOE (Ministry of Education in China) Project of Humanities and Social Sciences (No. 16YJC880057) and the Fundamental Research Funds for the Central Universities (No. 201564041). 


\section{Introduction}

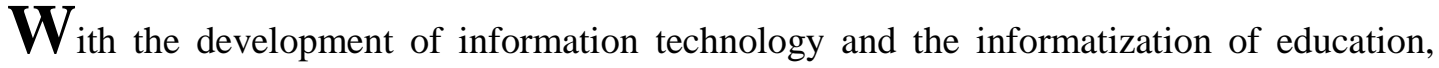
instructors have created countless learning resources. It has been a difficult task for learners to choose appropriate learning resources from the Internet in order to achieve established learning goals [1]. Without effective controls, extraneous cognitive resources will lead to learners' cognitive overload and significantly influence the outcomes from e-learning [2]. Therefore, learning systems need to realize the function that recommends learning resources based on distinct learners [3].

A learning path can be defined as a series of learning units leading to a particular learning outcome [4]. It is hardly an impossible task to design a single learning path to meet every learner's requirements [5]. An adaptive learning path is created and developed in this context. It is one of the implementation approaches of adaptive learning, which dynamically provides learning resources according to each learner's preferences and needs $[6,8]$. The major consideration for adaptive learning is the suitability between the learners and the learning resources [9]. Researchers have studied the various factors that influence suitability [3, 10, 11]. In their studies, multiple factors were simultaneously taken into account. Therefore, the essence of adaptive learning is a multi-objective optimization problem. The ultimate aim of the adaptive learning path is finding the optimal path through massive learning resources [7]. In this way, the "information overload" and "information confusion" problems in e-learning can be solved.

The basic structure of the adaptive learning path recommendation system includes the knowledge construct, user model, media space, and adaption model [9]. The media space and user model are the components for learning resources and learners. They are the bases for this system $[15,16]$. The adaption model defines the knowledge and content selection algorithms in which machine learning algorithms have been widely applied [7, 17]. The knowledge construct is the representation of the domain knowledge that contains concepts and their relationships. In general, the adaptive learning path should fulfil the following requirements: the knowledge construct should balance the domain knowledge with the learner's prior knowledge, the concepts should be imparted in the correct order, and the selected LOs should meet the learner's characteristics. In previous studies $[3,7,12,16]$, LO selection and sequencing have drawn great attention, while the concepts have been ignored. The reason for that is that the concept and its relationships are included in the LOs. However, large similar LOs are created for the same concept at present. Using the concepts initially rather than the LO directly can vastly decrease the complexity of this problem.

The paper is a cross study of computer applications, artificial intelligence and instructional technology in which an efficient approach for adaptive learning path recommendation is proposed. Firstly, a concept map is created using graph theory. Then, the map is traversed by the defied rules, and the adaptive learning path recommendation is converted to a multiobjective optimization problem. By introducing the ideas of prey and following the fish swarm algorithm, an improved immune algorithm is proposed to solve the problem. Our goal is to provide a personalized learning path for each learner and then improve their learning outcomes. The paper further explores sophisticated evolutionary algorithms' applications and accelerates the development of artificial intelligence in the educational domain. The remainder of the paper is organized as follows. Section 2 explores the related works and the relationships between them and our research. Section 3 provides a detailed description of the adaptive learning path recommendation approach and designs a web-based learning system based on the proposed approach. In section 4 , several simulation experiments 
are carried out to evaluate our approach from a computational perspective. Then, in section 5, an empirical study is also carried out to validate our approach from a pedagogical view. In the last section, the conclusions and the directions of future studies are proposed.

\section{Related Work}

Durand et al. define a learning path as an itinerary connecting several LOs [12]. Based on graph theory, they designed a recommendation system considering the competency dependencies among LOs [12]. Graph theory is a mathematical description of objects and their relationships. It has been successfully applied to solve many educational problems. Alian et al. constructed a direct acyclic graph from the relations among LOs for visualization, which are inherited from knowledge and the media space [20]. Alshalabi et al. modelled e-learning systems as a weight directed graph where each node represents a course unit [21]. Another important application scenario is creating a concept map that represents the knowledge structure of a field without losing the essential concepts and relationships [22]. However, it has been a common problem in previous studies that concept maps are predefined and adapted to a specific field [3, 9]. As a result, the individuals' knowledge structure is ignored. Some learners may not have the basic ability to understand the basic concepts. Therefore, it is very necessary to use graph theory to dynamically create learner-centred concept maps.

Evolutionary algorithms are another useful tool for tackling the adaptive learning path recommendation, in which it is seen as a combinatorial optimization problem [3]. Chen proposed a genetic-based personalized learning path generation algorithm to meet specific individual needs [10]. Another research used the enhanced genetic algorithm (GA) with TOPSIS to facilitate the search for a near optimal learning path [16]. Although GA is one of the most popular evolutionary algorithms, it has some disadvantages such as prematuration and stagnation. Other sophisticated evolutionary algorithms are also applied to solve this problem. Kurilovas et al. applied the ant colony optimization (ACO) method to generate a learning path based on students' learning styles, which observably improved students' learning outcomes and saved their learning time [24]. Wan \& Niu proposed a learner oriented learning recommendation approach based on mixed concept mapping and an immune algorithm (IA) [3]. IA is an algorithm that mimics the biological immune system, where the antigens and the antibodies are equivalent to the objective function and the feasible solution in a conventional optimization approach. With the antibody diversity mechanism of an immune system, IA excels at avoiding the prematuration phenomena and getting the global optimization [26]. Wan \& Niu's experience has proved that IA is superior to GA, ACO and the particle swarm optimization (PSO) for this problem. By using some special information related to the problems, the evolutionary algorithms can control the process of searching the state space. At this stage, they are dominated by the imitation of nature and are also largely based on the stochastic search technique [27]. Many new algorithms are proposed to optimize the effect $[28,29]$. The evolutionary algorithm is still a hot research field.

\section{Methods}

The adaptive learning path recommendation is divided into two parts in this paper: knowledge organization and curriculum design. The knowledge organization includes the process of organizing concepts that learners need to master. The curriculum design is the process of picking LOs from the repository to formulate an optimal learning path. The steps of our algorithm are shown as Fig. 1. The concept repository is supposed to be extremely large so 
that the concepts that learners need cannot be sequentially discovered. First, using graph theory, irrelevant concepts are eliminated to form a subgraph. The subgraph is actually a learner centred concept map established on the selected concepts and their relationships. Then, from the map, a traversal algorithm is used to generate a linear sequence following the prior knowledge relationships. At last, IIA is proposed to search for the optimal LO combination as the adaptive learning path based on the sequence.

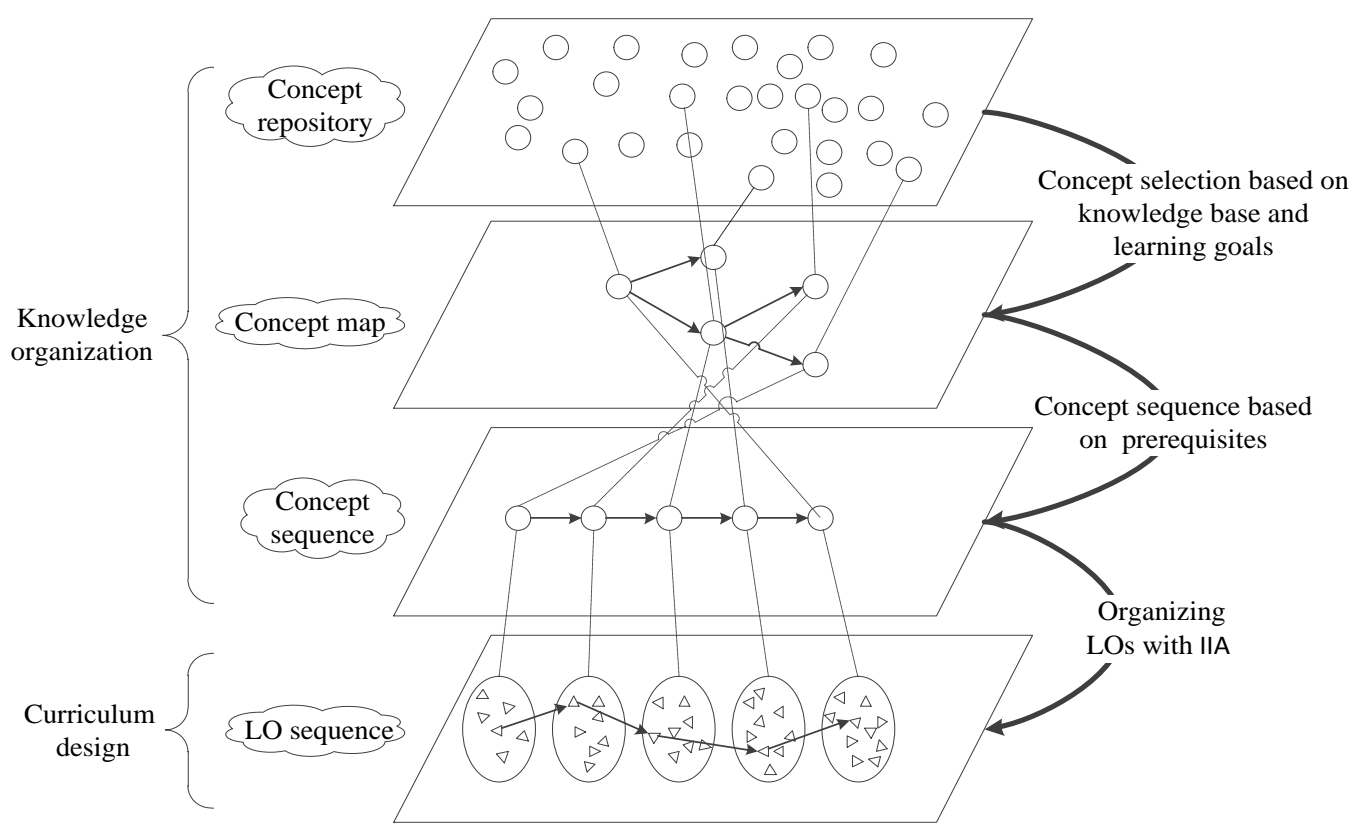

Fig. 1. The approach of adaptive learning path recommendation.

\subsection{Learner and learning object modelling}

There are many factors to consider for how to provide an adaptive learning path, such as attitude, motivation and so on [3]. Although more detailed information means better accuracy, in order to make the experiment more representative, the learning goals, the knowledge base, and the learning styles are the characteristics we selected. Actually, some related characteristics can be added to the model without much change. Because the system needs to select which concepts to impart, according to the principles of instructional design, it must get the knowledge base and learning goals for the current learner. In the meantime, it has been proved that there is a significant relationship between a learner's academic performance and learning style. The selection of learning objects that are appropriate to the individual's learning style characteristics will help improve the learning process and thus improve the learning quality. Therefore, we define a 3-tuple to represent the learner model, which is \{Learning Goal, Knowledge Base, Learning Style\}. However, the learner model can also include other factors such as motivation to establish a better match, which are treated similarly as the learning style. Theoretically, the factors are hard to exhaust. Learning style is a representative case. It is multi-dimensional, and so its match is a multi-objective problem.

The realization of learning goals needs the learners to master the necessary concepts. A Learning Goal is represented by $\left\{G_{1}, G_{2}, \ldots, G_{n}\right\}$, in which $G_{i}$ is a concept. The Knowledge Base is the concepts that the learner has mastered, and they are depicted as $\left\{B_{1}, B_{2}, \ldots, B_{n}\right\}$, in which $B_{i}$ is a concept too. The above characteristics are related to knowledge organization. 
The following characteristics influence the curriculum design. Each learner belongs to a particular learning style, which influences the learning approaches and strategies [11]. There are many mature models in the literature to describe learning styles, and it has been proven that the most popular learning style model is the Felder-Silverman Learning Style Dimensions [31]. The Felder \& Silverman model divides students' learning styles into four dimensions, and each has two categories. The four dimensions are Process (Active and Reflective), Input (Visual and Verbal), Perception (Sensitive and Intuitive) and Understanding (Sequential and Global) [32]. The results for the Felder \& Silverman learning styles are shown as Table 1, which is obtained from the Learning Styles Questionnaire. The questionnaire consists of 44 questions, each of which has two options: a and b. Each dimension of the learning style corresponds to 11 questions. In a dimension, assume that the total number of selected questions $\mathrm{a}$ is $\mathrm{M}$ and $\mathrm{b}$ is $\mathrm{N}$. The dimension's level is the bigger of $\mathrm{M}$ and $\mathrm{N}$. The smaller one is subtracted, and the category belongs to the bigger one. For example, if $\mathrm{M}=5$ and $\mathrm{N}=7$, the value of this dimension is $2 \mathrm{~b}$. The higher the level is, the higher the degree of belonging to its category. For convenient processing by computer, the value in each dimension is normalized, where $11 \mathrm{~b}$ is equal to 1 and $11 \mathrm{a}$ is equal to 0 . Therefore, the learning style is depicted as $\left\{\left(S T_{1}, e_{1}\right),\left(S T_{2}, e_{2}\right),\left(S T_{3}, e_{3}\right),\left(S T_{4}, e_{4}\right)\right\} . S T_{i}$ represents one dimension of the learning style. $e_{i}$ represents the value of dimension $S T_{i}, e_{i} \in[0,1]$.

Table 1. Felder \& Silverman learning style results

\begin{tabular}{cccccccccccccc}
\hline \multicolumn{11}{c}{$\leftarrow \mathrm{a}$} & \multicolumn{11}{c}{$\mathrm{b} \rightarrow$} \\
\hline ACT & 11 & 9 & 7 & 5 & 3 & 1 & 1 & 3 & 5 & 7 & 9 & 11 & REF \\
SEN & 11 & 9 & 7 & 5 & 3 & 1 & 1 & 3 & 5 & 7 & 9 & 11 & INT \\
VIS & 11 & 9 & 7 & 5 & 3 & 1 & 1 & 3 & 5 & 7 & 9 & 11 & VRB \\
SEQ & 11 & 9 & 7 & 5 & 3 & 1 & 1 & 3 & 5 & 7 & 9 & 11 & GLO \\
\hline \hline
\end{tabular}

The LO model is a structured response to the above learner model. We define a 2-tuple to epitomize the LO's characteristics with \{Concept, Learning Style\}. The concept represents the concept that is the basis of the LO's design. In this paper, we assume that an LO is created in response to the signal concept included in the concept repository. It has been covered that learners with different learning styles will have different processing, perceptions, receptions, and information comprehension. Therefore, some standards have been established when creating learning resources for learners with different learning styles [15, 32, 33]. Corresponding to the learner's learning style, the Learning Style of the LO model is depicted as $\left\{\left(S T_{1}, e_{1}\right),\left(S T_{2}, e_{2}\right),\left(S T_{3}, e_{3}\right),\left(S T_{4}, e_{4}\right)\right\} . S T_{i}$ represents one dimension of the learning style. $e_{i}$ represents the value in dimension $S T_{i}, e_{i} \in[0,1]$.

Moreover, a relation matrix is created to represent the relationships among LOs, where the columns and rows represent LOs and the elements represent the relationships. According to the strength of the relationship, the element is assigned a value between 0 and 1 . If the two LOs are totally independent, the element is 0 . If the two LOs are entirely interdependent, the element is 1.

\subsection{Knowledge organization}

\subsubsection{Concept modelling}

In this paper, the concept is seen as the framework to construct learning paths. It is necessary to design a concept model to organize and manage concepts. Lin, Lin, \& Huang emphasized that prior knowledge is essential in learning new concepts [34]. Therefore, the concept model 
contains a unique identifier in the concept repository and prior knowledge relationships. The concept model is shown in Fig. 2, in which the identifier is represented by a letter. Concept $A$ has two prerequisite concepts, $C$ and $D$. In addition, concept $A$ is the prerequisite concept of $B$.

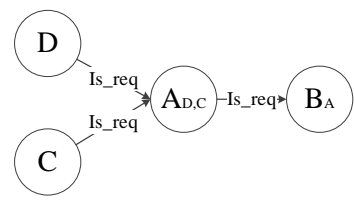

Fig. 2. The description of the concept model

Based on the designed concept model, the concept repository can be represented by a directed graph, $G=(V, E)$, which consists of the following:

- A non-empty finite set $V$ of the concepts, which are called nodes, and

- A finite set $E$ of the relationships among the concepts, which are called edges.

\subsubsection{Concept map creation}

In the learning process, learners with different knowledge bases and learning goals need to cover different concepts [41]. Furthermore, the knowledge organization has to consider the relationships between the concepts. To meet the requirements above, we use Durand's [12] algorithm to get a subgraph to form the large concept repository. Actually, the subgraph is a learner-centred concept map. This algorithm can be seen as the iteration generating sets. It begins with the learner's learning goal $\beta$ and ends with learner's knowledge base $\alpha$. The detail is shown below:

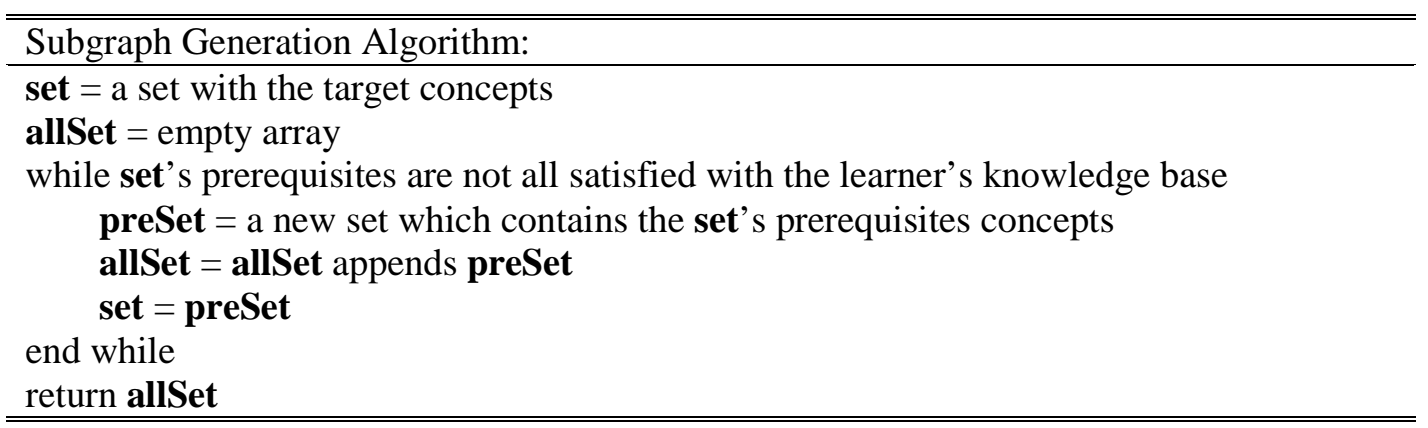

It will not stop until the prerequisites of all sets have been mastered by the learner. In the case of the three sets Set1 to Set3, as illustrated by Fig. 3, Set1 will be first considered, followed by Set2 and then Set3.

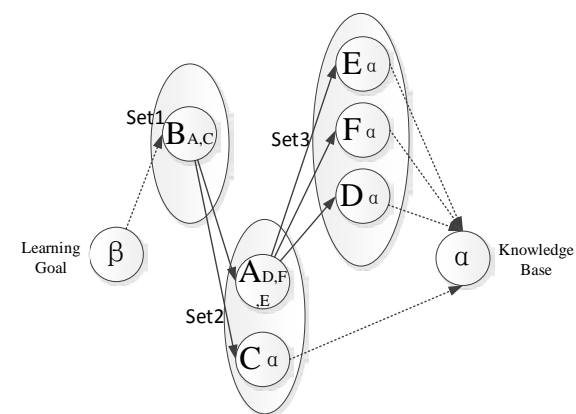

Fig. 3. Subgraph generation algorithm 
The knowledge structure of a discipline can be divided into a series of concepts [35], and so it is feasible to create a concept map to describe the knowledge structure. Moreover, the interdisciplinary knowledge involves an interconnection of the basic knowledge from different disciplines [36]. The interdisciplinary knowledge structure can be automatically constructed using this algorithm when given the intersections. Therefore, the knowledge structure becomes scalable, flexible, and extensible.

\subsubsection{Concept sequence generation}

No matter whether they are under traditional or computer assisted instruction, the process of learning concepts should be specified in the proper linear sequence $[41,42]$. In other words, it should follow the didactical precedence relationships among concepts. In the process, maintaining the prior knowledge relationships with concept maps is the main concern. Moreover, relevant concepts should be placed together so that learners can establish connections among them [41]. The proposed traversal algorithm starts randomly with the concept whose prerequisites are included in the learner's knowledge base. When the current branch has been traversed, the branch that supports the same advanced concept is selected to continue. Later, with the running of the algorithm, all underlying concepts will have been traversed. Therefore, the advanced concepts become accessible. The detail is shown below:
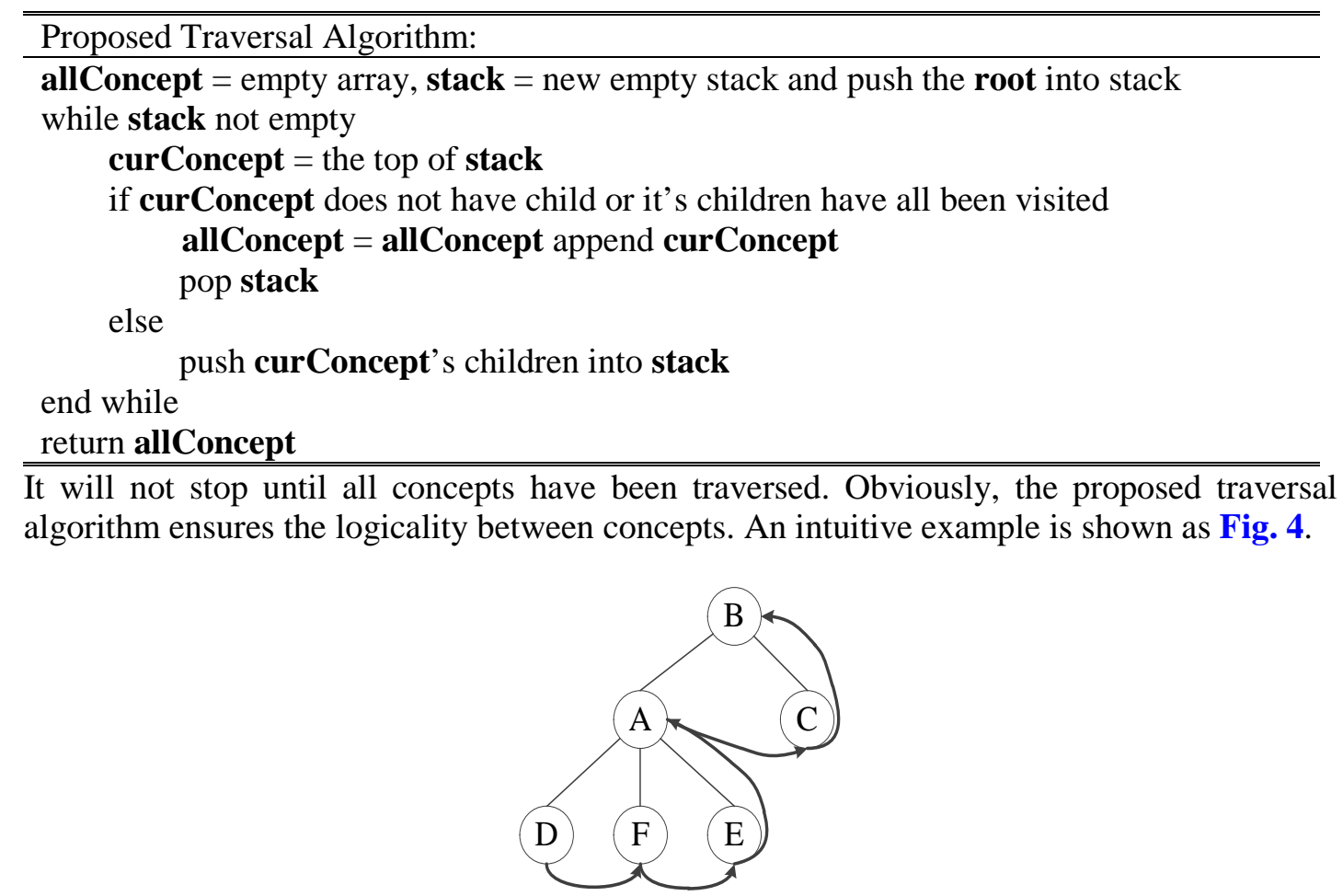

Fig. 4. Proposed traversal algorithm

\subsection{Curriculum design}

Through previous work, the learner's requisite concepts are selected and sorted in order. It is time to prepare the concrete learning materials. Assuming that each concept includes $P$ LOs and one LO must be selected for the concept, in order to generate the optimal learning path with $N$ concepts, there are still $P^{N}$ solutions need that to be tested. Therefore, the adaptive 
learning path recommendation is classified both as a multi-objective combinatorial optimization problem and an NP-hard problem [14]. It will consume much time to search for the optimal learning path. In this paper, an improved immune algorithm based on the Artificial Fish Swarm Algorithm (AFSA) is proposed to solve the combinatorial optimization problem of curriculum design. The flowchart of the IIA is shown in Fig. 5.

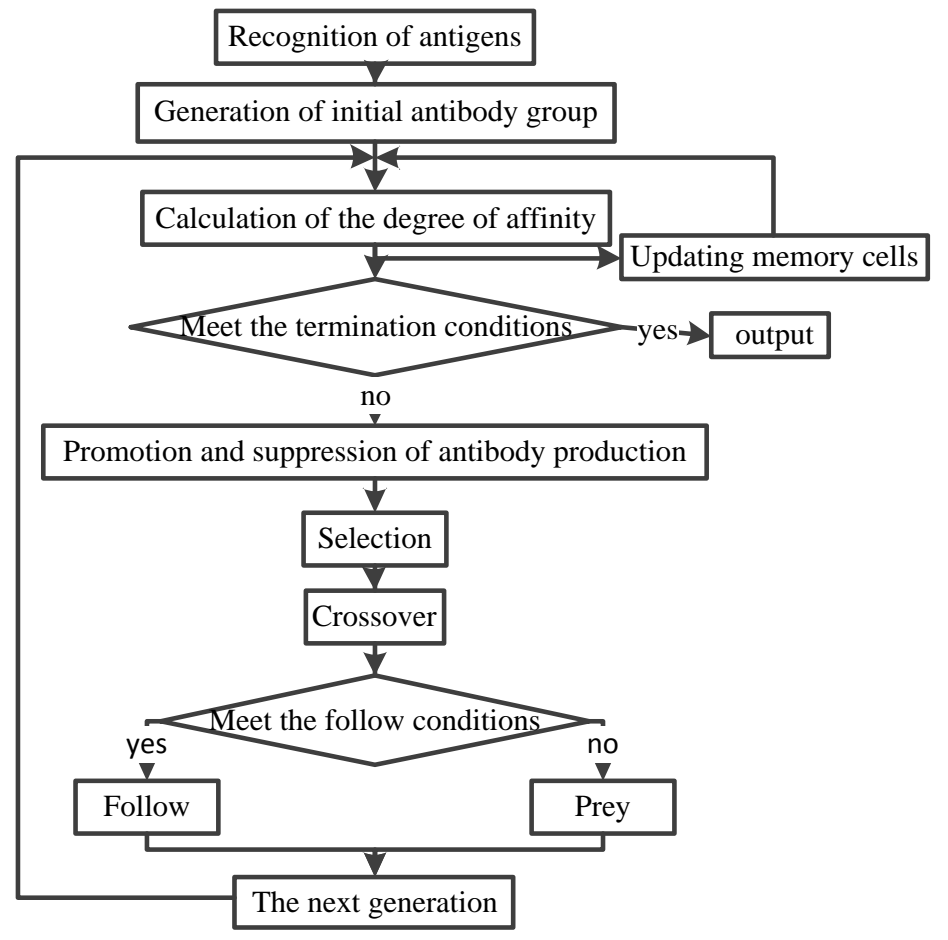

Fig. 5. The flowchart of the IIA

\subsubsection{Recognition of antigen}

In the IA, the problem to be solved is regarded as the antigen, and the antibody is seen as the solution. Under the condition that the concept sequence has been created in the previous section and there are multiple candidate LOs for each concept, the crux of generating the adaptive learning path is selecting an $\mathrm{LO}$ for each concept to generate an optimal learning path. Moreover, multiple objectives should be considered in this process.

\subsubsection{Antibody encoding}

To be clear, the LOs are grouped by their corresponding concepts, which are shown in Fig. 6. The GroupK $P_{i}$ includes the $L O_{i, j}$ corresponding to the ith concept, where the combination of $i$ and $j$ uniquely represent one LO. A chromosome frame is obtained by exhausting the LOs whose concepts are included in the concept sequence. If the locus is encoded as 0 , it means that the corresponding LO is not contained in this antibody. Otherwise, if the locus is encoded as 1 , it means that the corresponding LO is contained in this antibody. An example is shown in Fig. 7. 


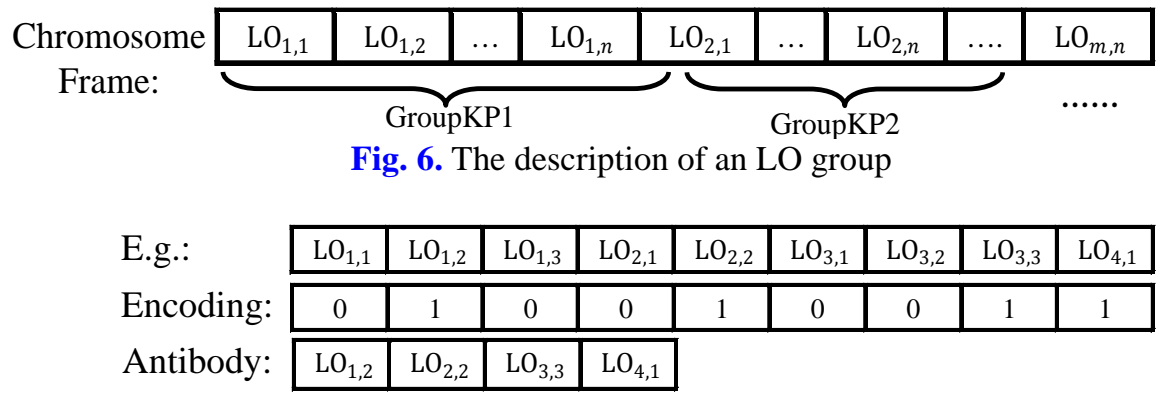

Fig. 7. Chromosome frame and antibody encoding

\subsubsection{Initialization antibody group}

To meet the requirements of the optimal learning path, there is a particular rule to be followed when generating an antibody. Each concept group should choose exactly one LO so that these learning resources can instruct the learners to master the necessary concepts and minimize their cognitive overload simultaneously. Because the LO sequence is inherited from the concept sequence, the didactical precedence relationships among concepts are preserved, which means that the prior knowledge will be learned in advance. One hundred antibodies are randomly generated as an initial population according to this rule.

\subsubsection{Affinity calculation}

The affinity in IA consists of two parts: the affinity between the antibody and antigen and the affinity among antibodies. It combines the antibody's fitness with the diversity. The first part represents the antibody's degree of antigen recognition, which is called its fitness. It is defined as

$$
A_{v}=1 / F_{v}
$$

where $A_{v}$ defines the affinity between $V$ antibody and antigen. $F_{v}$ is the general objective function. Assuming that $M$ concepts are chosen, the learning path can be expressed as $B, B=\left\{B_{1}, B_{2}, \ldots, B_{M}\right\}$, where $B_{i}$ is the ith LO. $U$ represents the learner model. $w_{i}$ denotes the set of objective weights. $n$ is the number of optimization objectives. In this study, $n=2$, which means that two objectives are considered. $f_{i}$ is a penalty function. As a result, the aim is to search for the minimum solution of the objective function, which is shown as follows:

$$
\begin{aligned}
F= & \min \left(\sum_{i=1}^{n} w_{i} f_{i}(U, B)\right) \\
\text { s.t. } & B_{i} \in G \operatorname{GroupKP} . \quad i=1,2, \ldots, M \\
& \sum_{i=1}^{n} w_{i}=1
\end{aligned}
$$

Next, according to previous studies $[3,11,16]$, two optimization objectives will be introduced in detail. $f_{1}$ is the LOs' corresponding characteristics that should satisfy the learner's learning style. It is computed as

$$
f_{1}=\frac{1}{M} \sum_{i=1}^{M} \frac{\sum_{j=1}^{k}\left|U L S_{j}-L L S_{i, j}\right|}{k}
$$

where $k$ is the dimension of the learning style. In the Felder \& Silverman learning style, $k=$ 4. $U L S_{j}$ defines the learner's learning style in the $j$ th dimension. $L L S_{i, j}$ defines the corresponding characteristics of the ith $\mathrm{LO}$ in the jth dimension. $f_{2}$ is the $\mathrm{LO}$ combination that 
leads to an awful learning output that should be avoided. It is computed as

$$
f_{2}=\frac{2 \sum_{i=1}^{M-1} \sum_{i=i+1}^{M} R_{L_{i}, L_{j}}}{M(M-1)}, R_{L_{i}, L_{j}}=\frac{N\left(L_{i} \cap L_{j}\right)}{N\left(L_{i}\right)}
$$

where $R_{L_{i}, L_{j}}$ is the correlation strength of $L_{i}$ and $L_{j}, N\left(L_{i}\right)$ is the number of previous learners who fail in $L_{i}$ and $N\left(L_{i} \cap L_{j}\right)$ is those who fail in both $L_{i}$ and $L_{j}$.

The foundation of calculating the affinity among antibodies is identifying the affinity between two antibodies. In this paper, it is measured by Forrest's R consecutive matching method [38]. It is defined as

$$
S_{v, s}=k_{v, s} / L
$$

where $S_{v, s}$ defines the affinity between $V$ and $S, L$ defines the length of the antibody, and $k_{v, s}$ defines the number of same bits. An example is shown in Fig. 8 in which the length of the antibody is 9 and the second, the fifth, and the sixth are the same bits. Therefore, $k_{v, s}=3$, $L=9$ and $S_{v, s}=1 / 3$.

$$
\begin{aligned}
& \mathrm{V}: \begin{array}{|l|l|l|l|l|l|l|l|l|}
\hline \mathrm{LO}_{1,2} & \mathrm{LO}_{2,2} & \mathrm{LO}_{3,2} & \mathrm{LO}_{4,5} & \mathrm{LO}_{5,9} & \mathrm{LO}_{6,3} & \mathrm{LO}_{7,2} & \mathrm{LO}_{8,2} & \mathrm{LO}_{9,2} \\
\hline
\end{array} \\
& \mathrm{S}: \begin{array}{|l|l|l|l|l|l|l|l|l|}
\hline \mathrm{LO}_{1,1} & \mathrm{LO}_{2,2} & \mathrm{LO}_{3,8} & \mathrm{LO}_{4,7} & \mathrm{LO}_{5,9} & \mathrm{LO}_{6,3} & \mathrm{LO}_{7,9} & \mathrm{LO}_{8,1} & \mathrm{LO}_{9,3} \\
\hline
\end{array}
\end{aligned}
$$

Fig. 8. Affinity between antibodies

The affinity among antibodies is the proportion of antibodies that are similar to a specific antibody in the population. Actually, it is the concentration of similar antibodies, which indicates the diversity of the population. It is computed as

$$
C_{v}=\sum_{s=1}^{N} S_{v, s} / N, \mathrm{~S}_{v, s}= \begin{cases}1 & \mathrm{~S}_{v, s}>T \\ 0 & \text { otherwise }\end{cases}
$$

where $C_{v}$ represents the concentration of antibody $V$, the size of the antibody population is $N$, and $T$ is the threshold value that decides whether $V$ is similar to $S$.

\subsubsection{Updating memory cells}

To ensure a rapid and stable convergence to the global optimal solution, the memory cell strategy is introduced, where the highest affinity solutions are selected as memory cells. As a supplement, the elitist strategy is adapted to preserve the best fitness solutions. In each iteration, part of the highest affinity and best fitness antibodies are selected to update the memory cells.

\subsubsection{Selection}

Selection is the operator through which well-behaved antibodies are selected to generate the next generation. The expected reproduction rate of an individual is determined by $A_{v}$ and $C_{v} . P_{v}$ represents the expected reproduction rate of antibody $V . \alpha$ is a constant. It is shown as follows:

$$
P_{v}=\alpha \frac{A_{v}}{\sum_{i=1}^{N} A_{i}}+(1-\alpha) \frac{C_{v}}{\sum_{i=1}^{N} C_{i}}
$$

The selection probability (denoted by $S P_{v}$ ) is obtained by the expected reproduction rate. It is computed as 


$$
S P_{v}=\frac{P_{v}}{\sum_{i=1}^{N} P_{i}}
$$

We employ the roulette wheel selection to get the item. It is shown in Fig. 9. A larger expected reproduction rate means a larger occupied area in the pie chart, and so the corresponding antibody has a larger selection probability. Moreover, the antibodies can be repeatedly chosen in this operator.

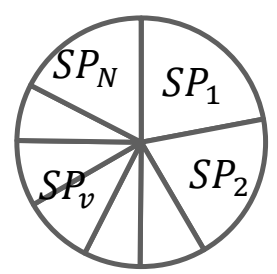

Fig. 9. Roulette wheel selection

\subsubsection{Crossover}

The crossover operator can generate the new learning path. It aims to increase the diversity of the population. In the crossover operator, two antibodies are selected as parents according to the crossover probability. The one-point crossover strategy is adopted in this part. Specifically, one crossover point is randomly selected. The offspring are generated by exchanging the whole sequence after this point. An example is shown in Fig. 10.

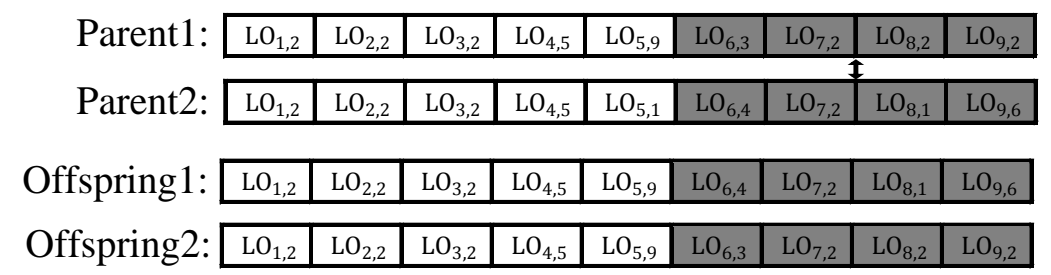

Fig. 10. One-point crossover strategy

\subsubsection{Prey and follow}

In the standard IA, the mutation operator changes the antibody according to a fixed mutation rate. It aims to increase the diversity of population. Obviously, the population information is ignored here. AFSA is one of the most appropriate approaches for swarm intelligence optimization, and it is inspired by the movement of fishes [37]. The basic behaviours of artificial fish in the AFSA include prey, swarm, follow, move and leap. In order to make up for its shortcomings, the follow and prey operators of the AFSA are introduced into the IA.

\begin{tabular}{|c|c|c|c|c|c|c|c|c|c|}
\hline \multirow{3}{*}{ Parent: } & $\mathrm{LO}_{1,1}$ & $\mathrm{LO}_{1,2}$ & $\mathrm{LO}_{1,3}$ & $\mathrm{LO}_{2,1}$ & $\mathrm{LO}_{2,2}$ & $\mathrm{LO}_{3,1}$ & $\mathrm{LO}_{3,2}$ & $\mathrm{LO}_{3,3}$ & $\mathrm{LO}_{4,1}$ \\
\hline & 0 & 1 & 0 & 0 & 1 & 1 & 0 & 0 & 1 \\
\hline & \multicolumn{3}{|c|}{$\mathrm{LO}_{1,2}$} & \multicolumn{2}{|c|}{$\mathrm{LO}_{2,2}$} & \multicolumn{3}{|c|}{$\mathrm{LO}_{3,1}$} & $\mathrm{LO}_{4,1}$ \\
\hline \multirow{3}{*}{ Offspring: } & $\mathrm{LO}_{1,1}$ & $\mathrm{LO}_{1,2}$ & $\mathrm{LO}_{1,3}$ & $\mathrm{LO}_{2,1}$ & $\mathrm{LO}_{2,2}$ & $\mathrm{LO}_{3,1}$ & $\mathrm{LO}_{3,2}$ & $\mathrm{LO}_{3,3}$ & $\mathrm{LO}_{4,1}$ \\
\hline & 0 & 1 & 0 & 0 & 1 & 0 & 0 & 1 & 1 \\
\hline & \multicolumn{3}{|c|}{$\mathrm{LO}_{1,2}$} & \multicolumn{2}{|c|}{$\mathrm{LO}_{2,2}$} & \multicolumn{3}{|c|}{$\mathrm{LO}_{3,3}$} & $\mathrm{LO}_{4,1}$ \\
\hline
\end{tabular}

Fig. 11. Prey operator 
The prey operator imitates the basic biological behaviour of seeking food. It contains the following manipulations. It randomly selects a mutation point and replaces the $\mathrm{LO}$ in the mutation point with a new LO. The new LO is randomly selected, but it must belong to the same concept group as the previous one targeting the current problems. The prey operator can explore the solution space and retain the diversity of the population, which is shown in Fig. 11. Artificial fish will move to their best neighbour when their surroundings are not crowded in the follow operator, which can accelerate the movement to the global extreme value field from the local extreme values. Vision represents a certain distance. If the distance between two fishes is less than it, the two fishes are called neighbours, as shown in Fig. 12. The distance between fishes is calculated as

$$
\operatorname{Dist}(S, V)=\sum_{i=1}^{M} H_{i}, \quad\left\{\begin{array}{l}
\text { if } \mathrm{S}_{i}=V_{i} \mathrm{H}_{i}=0 \\
\text { if } \mathrm{S}_{i} \neq V_{i} \mathrm{H}_{i}=1
\end{array}\right.
$$

where $S_{i}$ and $V_{i}$ represent the ith bits on the antibody $S$ and the antibody $V$. The crowd factor $\delta$ is used to restrict the scale of fish. If $Y_{\min } * n<Y_{i} * \delta$, it means that the best neighbour has a higher performance than the current individual and the degree of crowding by neighbours is acceptable. Therefore, the individual will move to the best neighbour. That is, the element from the best neighbour is copied into the current individual. Otherwise, the individual will be processed by the prey operator. Here, $Y_{\min }$ is the best neighbour's fitness, $n$ is the neighbourhood number, and $Y_{i}$ is the fitness of the current individual.

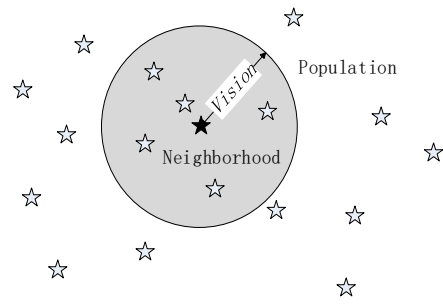

Fig. 12. The description of artificial fish's vision

In the initial stage, the distribution of the population is sparse. It means that individuals seldom have neighbours, and so the IIA can explore the solution space sufficiently, which guarantees the stronger global optimization capability. Then, the population gradually gathers at several excellent regions. The IIA could escape from the local optimum and accelerate the convergence speed to the globe optimum through the follow operator. During the posterior of the evolutionary process, the concentration of excellent individuals will increase and the diversity of the population will hastily decrease. However, the population's predation capacity will be enhanced under this condition, which ensures the sustained optimization ability of the algorithm. After analysing the principles of the IIA, the conclusion can be drawn that the improvements make the IA become more adaptive.

\subsection{System design}

A web-based learning system based on the proposed approach is designed to realize adaptive learning. As shown in Fig. 13, there are two types of users in this learning system: learners and instructors (experts). 


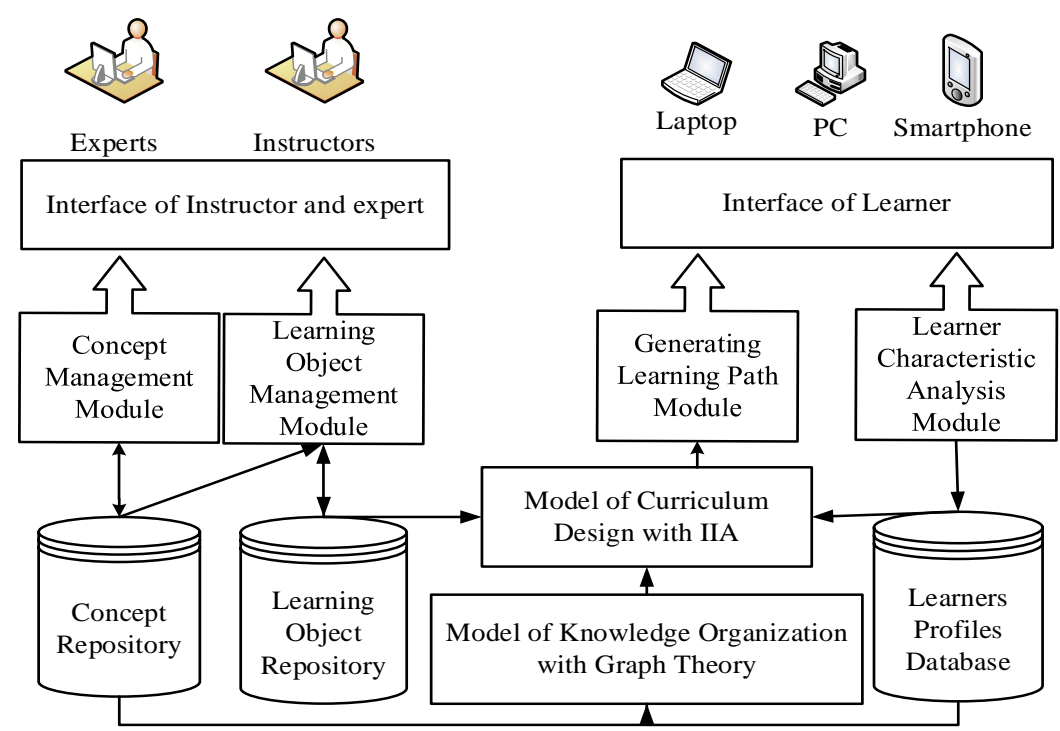

Fig. 13. Learning system model

By using the concept management module, instructors could manage the concept repository. Updating concepts and their relationships constantly is beneficial for the application scope and knowledge update of the learning system. The learning object management module is responsible for LO's management. Through the evaluation standards, the instructors could assign values to LO's characteristics and establish the corresponding relationships between LOs and concepts. The learner attribute analysis module is responsible for collecting learner characteristics. By combining the profile and concept repositories, the knowledge organization module using graph theory and the proposed traversal algorithm generates a concept sequence. In the curriculum design module, based on the above concept sequence, an adaptive learning path is picked from the LO repository employing the IIA. Eventually, LOs are presented to leaners through learning terminals according to the learning path. On one hand, these learning terminals deliver the teaching information. On the other hand, the data generated during the learning process could be collected through an equipment, which could improve the adaptability of the learning system.

\section{Computational experiments}

In this section, simulation experiments are carried out to validate the proposed approach's optimization ability with respect to its efficiency and effectiveness. As controlled trials, there are four approaches that have been validated. The first one generates a learning path randomly. It is fast and simple, but it has large stochastic volatility. The second one is a GA-based algorithm, TOPSIS, which was proposed by Lin et al. [16]. The next one is MCPIA [3]. It is an IA-based approach proposed by Shanshan Wan et al. The last one is ours. We use the same learner, learning object and concept models for the four methods. Each algorithm runs 20 times in the simulated dataset to reduce the randomness of the algorithm's implementation. As for the experimental environment, these approaches are implemented in MATLAB R2012a and run on a PC equipped with a 3.70GHz Intel i3-4170 CPU and 4GB RAM.

\subsection{Datasets}

Nine simulated datasets of the concept and LO repositories are generated. The simulated 
datasets are listed in Table 2. The numbers of concepts in the nine simulation datasets include 20, 50, and 100 while the numbers of LOs for each concept include 10, 30, and 50 . Furthermore, each LO contains the degree of dependence with other LOs and the four respective learning style characteristics of Process, Input, Perception, and Understanding. These characteristics are assigned with normalized values following a normal distribution. For the sake of simplicity, all the learning style characteristics of the learner instances are initialized to 0.5 .

Table 2. Simulated data set

\begin{tabular}{ccc}
\hline Data set & Number of Concepts & Number of LOs for each concept \\
\hline 1 & 20 & 10 \\
2 & 20 & 30 \\
3 & 20 & 50 \\
4 & 50 & 10 \\
5 & 50 & 30 \\
6 & 50 & 50 \\
7 & 100 & 10 \\
8 & 100 & 30 \\
9 & 100 & 50 \\
\hline
\end{tabular}

\subsection{Parameter Settings}

The parameter setting plays an important part in evolutionary algorithms' running performance [39]. The parameters of our approach are configured in this section. The IIA contains seven main parameters (i.e., the crossover probability, the mutation probability, the population size, the memory cells size, the immune balance factor $\alpha$, the vision range, and the crowd factor $\delta$ ). The initial values of the parameters are determined based on the previous studies [26, 37]. After using a one-at-a-time approach, the parameter values that lead to the best results are chosen. The crossover and mutation probabilities are set to 0.5 and 0.4 , respectively, which means that the selected antibodies have a 50 percent chance of crossover and a 40 percent chance of mutation. The population size is assigned as 100 and only one in ten antibodies are selected as memory cells. The immune balance factor $\alpha$ is set to 0.8 , which means that the affinity between the antibody and antigen has a greater influence than the antibody concentration here. The vision range is one tenth of the number of concepts.

\subsection{Performance analysis}

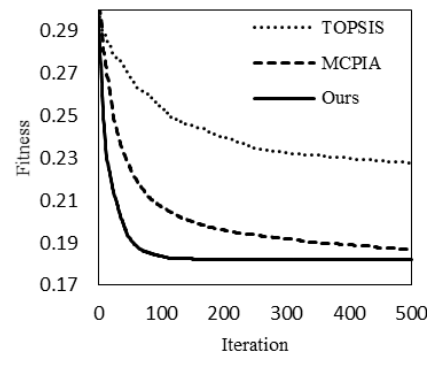

a

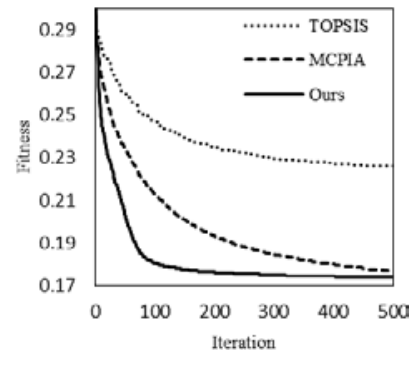

b

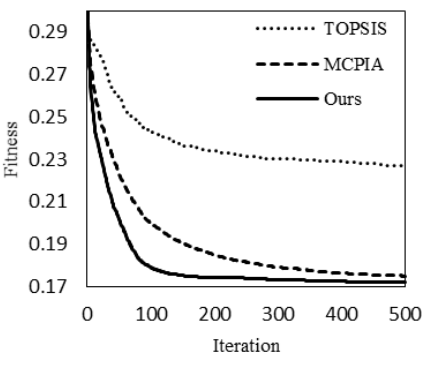

C

Fig. 14. Comparison of the convergence process with three approaches in the simulation datasets.

(a) Set 4. (b) Set 5. (c) Set 6. 
Fig. 14 shows the convergence curves of TOPSIS, MCLIA, and our method over 500 generations of the evolutionary process. The iteration number is the abscissa and the fitness is the ordinate in which a lower fitness value means that the solution is closer to the optimal. The scale of the experiment is to master 50 concepts. Fig. 14(a) is the convergence comparison chart for the 10 LOs for each concept, Fig. 14(b) is for 30, and Fig. 14(c) is for 50. Fig. 14(a) clearly shows the convergence of the three approaches' performances. Our approach converges much faster than MCPIA and TOPSIS, which can quickly converge to a global optimal with only 100 iterations. We can get the same conclusion from Fig. 14(b) and Fig. 14(c). From the results, we can conclude that our approach overcomes the shortcomings of premature convergence and stagnation and significantly increases the search efficiency. Therefore, the efficiency of our approach is better than TOPSIS and MCLIA for the current situation.

Table 3. Simulation experimental result

\begin{tabular}{|c|c|c|c|c|c|c|c|c|c|c|}
\hline \multicolumn{2}{|c|}{ Approach $\quad$ Dataset } & 1 & 2 & 3 & 4 & 5 & 6 & 7 & 8 & 9 \\
\hline \multirow{3}{*}{ RANDOM } & $\mathrm{AF}$ & 0.336 & 0.337 & 0.347 & 0.350 & 0.350 & 0.355 & 0.344 & 0.355 & 0.349 \\
\hline & $\mathrm{BF}$ & 0.285 & 0.286 & 0.280 & 0.319 & 0.314 & 0.337 & 0.330 & 0.340 & 0.321 \\
\hline & SD & 0.0249 & 0.0269 & 0.0354 & 0.0223 & 0.0210 & 0.0130 & 0.0109 & 0.0099 & 0.0139 \\
\hline \multirow{3}{*}{ TOPSIS } & AF & 0.214 & 0.197 & 0.202 & 0.228 & 0.226 & 0.227 & 0.255 & 0.250 & 0.250 \\
\hline & $\mathrm{BF}$ & 0.205 & 0.188 & 0.191 & 0.222 & 0.220 & 0.219 & 0.239 & 0.236 & 0.233 \\
\hline & SD & 0.0035 & 0.0049 & 0.0051 & 0.0031 & 0.004 & 0.004 & 0.0096 & 0.0082 & 0.0075 \\
\hline \multirow{3}{*}{ MCPIA } & $\mathrm{AF}$ & 0.176 & 0.149 & 0.152 & 0.187 & 0.177 & 0.175 & 0.198 & 0.190 & 0.187 \\
\hline & $\mathrm{BF}$ & 0.134 & 0.138 & 0.142 & 0.183 & 0.173 & 0.170 & 0.196 & 0.187 & 0.184 \\
\hline & SD & 0.0156 & 0.0062 & 0.0051 & 0.0055 & 0.0031 & 0.0032 & 0.0011 & 0.002 & 0.0017 \\
\hline \multirow{3}{*}{ Ours } & $\mathrm{AF}$ & 0.165 & 0.141 & 0.148 & 0.182 & 0.174 & 0.172 & 0.196 & 0.185 & 0.180 \\
\hline & $\mathrm{BF}$ & 0.120 & 0.124 & 0.135 & 0.174 & 0.170 & 0.168 & 0.194 & 0.182 & 0.178 \\
\hline & SD & 0.0153 & 0.0092 & 0.0061 & 0.0041 & 0.0022 & 0.0022 & 0.0011 & 0.0025 & 0.0013 \\
\hline
\end{tabular}

FA: Average fitness; FB: Best fitness; SD: Standard deviation

Table 3 shows the Average Fitness (AF), Best Fitness (BF), and Standard Deviation (SD) for these algorithms on the nine simulated datasets. BF is the optimal result of the 20 experiments, AF is the mean result of the 20 experiments, and SD indicates the fluctuation of the results of all 20 experiments. The numerical results in the table show that these three evolutionary algorithm based approaches significantly enhance the search performance compared with the random approach. Because the random approach has the worst $\mathrm{AF}$ and $\mathrm{BF}$ and more obvious fluctuations, the RANDOM based approach is a very bad choice for the learning path recommendation. Compared with the other evolutionary algorithm based approaches, our approach performs well on both indicators. It has the best fitness with good stability. In addition, the quality of the solutions found by our approach is always better than MCLIA, indicating that the results are improved by implementing the follow and prey operators. In summary, these results indicate that our approach is more effective. By avoiding the prematuration phenomena and enhancing the search ability in the solution space, it has the fastest convergence speed and a better global optimization ability. Therefore, our approach can get a satisfactory solution to problems with different scales, which satisfies the requirements of most real-life applications of adaptive learning path recommendations.

\section{Empirical study}

To verify the proposed approach from a pedagogical perspective, some learners are invited to test the proposed approach in a real-life situation. Using the Django framework [43], an Adaptive Learning System (ALS) is developed as the exp erimental platform with a 
browser-server architecture. Three recommendation approaches, including the proposed approach and the two approaches of Self-selected and TOPSIS, are selected for the control experiment.

\subsection{Experiment design}

A total of 90 healthy sophomores from Ocean University of China between the ages of 18 and 21 (mean age $=19.09$ ) voluntarily participated in the experiment. The number of males is 48 , and the number of females is 42 . They chose the same teacher's course and were divided into three equal classes. Their academic grades indicate that there are no significant differences among the classes. A Python programming course is taken as the learning resource. The course aims to guide the learner to master the basic python grammar and program design approach. The flip classroom is taken as the teaching form where the ALS administers the computer-based individual instruction outside the classroom. However, each class used a different recommendation approach based system and the teacher does not know it.

To explain how to perform the learning activities, we briefly introduce the ALS. Fig. 15(a) shows the layout of the concept management interface. As a teacher logs into this system, he can choose the course for which he is responsible and dynamically update, create or delete concepts on the left map. This ensures that each teacher can adjust the concept repository accordingly as needed inside the classroom. Fig. 15(b) displays a labelled learning object with its corresponding concept, learning style and other characteristics. Currently, the course materials are collected from publically accessible resources. Because the materials collected by the teacher are insufficient, there are four assistants involved in this activity. Fig. 15(c) shows the learner's profile. Among these characteristics, learning styles are generated by the system after filling out the Learning Styles Questionnaire. The learning goals and related knowledge base are chosen by learners from the concept map. When the personal information is confirmed, the adaptive learning path generation engine will work. Fig. 15(d) illustrates the generated learning path by the system. The concepts that the learner needs to master are placed in sequence on the left side. The recommended LO for each concept is displayed on the right side.

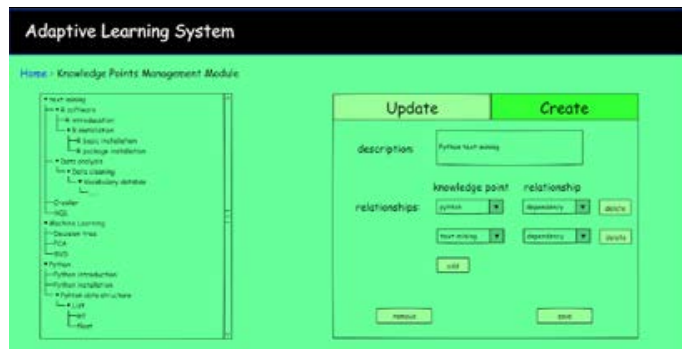

a

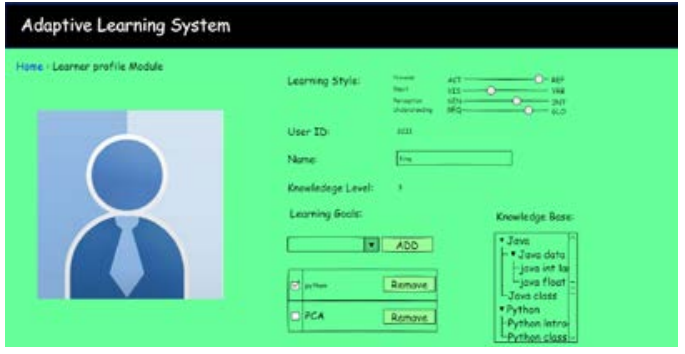

C

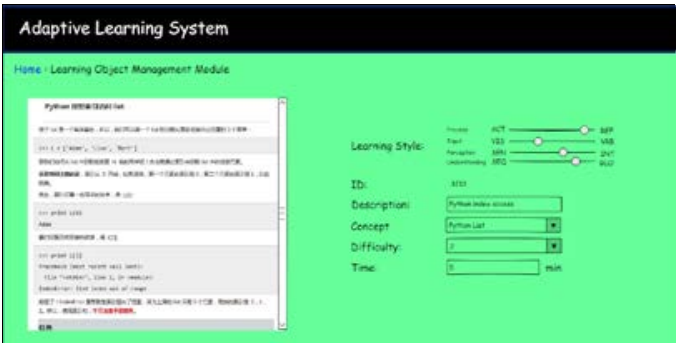

b

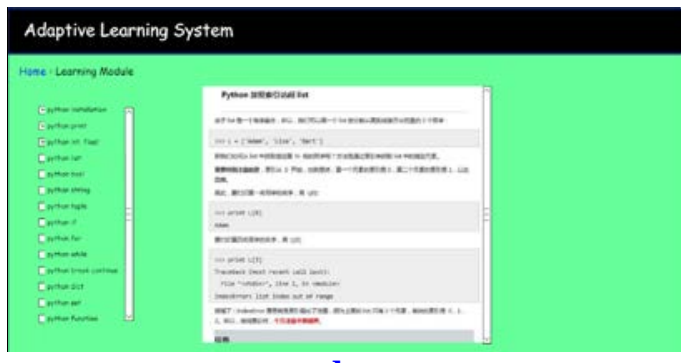

d

Fig. 15. Some screenshots from the ALS. (a) concept management interface, (b) learning object management interface, (c) a learner's personal centre, and (d) study interface 
The course ran for the spring term with two sessions per week. During the course, there are three integrated programming assignments related to specific themes. They are completed and submitted by the learner independently. The teacher has to evaluate the homework with a quantitative value varying from zero to five with one decimal place. Meanwhile, after using the computer-based individual instruction outside the classroom, the students were asked to express their satisfaction with the ALS using a Likert scale ranging from 1-5, where 1 denoted strong dissatisfaction and 5 indicated strong satisfaction. During the last week of the semester, a final exam was given to all participating students to evaluate the learning effect and it was graded by the teacher on a one hundred point scale.

\subsection{Result analysis}

In this section, the learners' homework, final exam grades, and satisfaction are analysed to evaluate the proposed approach. The mean value and standard deviation of each class are presented in Table 4. To analyse the results, the Student t-test for the comparison of two independent samples is performed many times. The Student t-test involves testing a null hypothesis against an alternative hypothesis. The null hypothesis means that there is no significant difference between the means of two independent samples, whereas the alternative hypothesis means that there is a significant difference between the means of two independent samples. In this work, all tests are two-tailed with a 5\% significance level and were performed using IBM SPSS Statistics 19 software. The corresponding t-statistics and P-values are shown in Table 5.

First, a t-test is performed using the homework grades data acquired during the integrated programming assignments to analyse whether there is a significant difference in the three classes. When comparing the classes using our approach with the self-selected and TOPSIS methods, the P-values are less than 0.05 , and therefore, the null hypothesis is rejected in favour of the alternative hypothesis. Thus, by combining their higher homework grades from Table 4, it can be concluded that the proposed approach can help learners produce better homework outcomes. Next, we analyse the exam grade using the same rules. It can be found out that there is no significant difference among TOPSIS and Our approach. However, the P-values between Self-selected and the two evolutionary algorithm based systems are both less than 0.05 , which means there is a significant difference. With respect to exam grades, generating the learning path based on an evolutionary algorithm is a good choice. When performing the t-tests using the satisfaction data, the P-values between our approach and the other two are both smaller than 0.05 . Therefore, the null hypotheses are rejected, indicating that the means of student satisfaction are different. As shown in Table 4, the results indicate that the mean satisfaction degree for the self-selected method is poor. This is logical since students have to manually find out what works best for them from lots of related contents. Meanwhile, the mean value of the satisfaction degree obtained by our approach is the highest. In aggregate, our approach is proved to be valuable in educational applications.

Table 4. Summary of the empirical study results

\begin{tabular}{|c|c|c|c|c|}
\hline \multicolumn{2}{|c|}{} & Self-selected & TOPSIS & Ours \\
\hline \multirow{2}{*}{ Homework Grade } & Mean & 3.68 & 4.13 & 4.56 \\
\cline { 2 - 5 } & SD & 0.57 & 0.52 & 0.30 \\
\hline \multirow{2}{*}{ Exam Grade } & Mean & 86.32 & 88.44 & 88.48 \\
\cline { 2 - 5 } & SD & 10.00 & 7.19 & 7.16 \\
\hline \multirow{2}{*}{ Satisfaction } & Mean & 3.01 & 4.10 & 4.49 \\
\cline { 2 - 5 } & SD & 0.57 & 0.41 & 0.40 \\
\hline
\end{tabular}

Mean: Mean value; SD: Standard deviation 
Table 5. t-statistic and P-values.

\begin{tabular}{|c|c|c|c|c|}
\hline \multicolumn{2}{|c|}{} & Ours \& Self-selected & Ours \& TOPSIS & TOPSIS \& Self-selected \\
\hline \multirow{2}{*}{ Homework Grade } & $\mathrm{t}$ & -7.470 & -3.923 & -3.186 \\
\cline { 2 - 5 } & P-value & 0.000 & 0.000 & 0.02 \\
\hline \multirow{2}{*}{ Exam Grade } & $\mathrm{t}$ & -3.512 & -1.103 & -2.598 \\
\cline { 2 - 5 } & P-value & 0.001 & 0.275 & 0.012 \\
\hline \multirow{2}{*}{ Satisfaction } & $\mathrm{t}$ & -11.653 & -3.701 & -8.552 \\
\cline { 2 - 5 } & P-value & 0.000 & 0.000 & 0.000 \\
\hline
\end{tabular}

\section{Conclusion and future work}

The adaptive learning path is an important research field in e-learning. It is quite necessary to discover the most efficient approach to realize it. This paper proposes an efficient approach based on graph theory and the IIA. Firstly, a learner-centred concept map is created according to the relationship between the learner model and prior knowledge. Then, a traversal algorithm is applied to form a linear concept sequence to maintain the didactical precedence relationships. Next, each concept in sequence is provided with an optimal LO to generate an adaptive learning path, which is converted to a multi-objective combinatorial optimization problem. The IIA is applied to facilitate the search for the near-optimal solution. We think that the recommended learning path can satisfy a learner's specific requirements and minimize his cognitive overload.

A series of simulation experiments are carried out to verify the efficiency and effectiveness of the proposed approach. In the field of solution quality, the proposed approach has the best fitness among the four approaches. It effectively enhances the global searching ability and avoids the prematuration phenomena. With respect to search efficiency, our approach can converge in fewer iterations in order to meet the precondition of excellent fitness. Consequently, the improved algorithm is able to efficiently solve the multi-objective combinatorial optimization problem. Moreover, a case study is also implemented to validate the proposed approach from a pedagogical point of view. Apart from the self-selected approach, the other two evolutionary algorithm based approaches are also applied for comparison. The results show that our approach achieves the best outcomes in terms of homework, final exam grades, and satisfaction.

The main contributions of this paper are as follows. This paper proposes an efficient approach for adaptive learning path recommendations and further explores sophisticated evolutionary algorithms' applications in the educational domain. The approach has clear levels and is well-targeted and expansible, and the details of it have also been explained, which makes it easy to implement. Second, an improved immune algorithm based on the artificial fish swarm algorithm is proposed to improve the optimization performance of the standard IA. By introducing the ideas of prey and follow from the AFSA, the performance of the standard IA is significantly improved. Moreover, this paper develops a learning system based on the proposed approach and meaningfully combines artificial intelligence and teaching.

However, we only take the learning goals, knowledge base, and learning styles as the bases to generate an adaptive learning path. There are more factors that can be taken into consideration in practical applications, such as the features of the learning environment and the needs of teachers, depending on the practice. The learning path recommendation problem still needs to be enhanced. In the future, we will keep improving our model to integrate the various needs. 


\section{References}

[1] H. Tseng, C. Chiang, J. Su, J. Hung and B.E. Shelton, "Building an Online Adaptive Learning and Recommendation Platform,” in Proc. of Int. Symposium on Emerging Technologies for Education, pp. 428-432, 2016. Article (CrossRef Link).

[2] J.J. Jeroen, van Merriënboer and P. Ayres, "Research on cognitive load theory and its design implications for e-learning,” Educational Technology Research and Development. Vol. 3, no. 3, pp. 5-13, 2005. Article (CrossRef Link).

[3] S. Wan and Z. Niu, "A learner oriented learning recommendation approach based on mixed concept mapping and immune algorithm,” Knowledge-Based Systems, Vol. 103, pp. 28-40, 2016. Article (CrossRef Link).

[4] J. Janssen, A.J. Berlanga and R. Koper, "Evaluation of the Learning Path Specification," Educational Technology \& Society, Vol. 14, no.3, pp. 218-230, 2011. Article (CrossRef Link).

[5] C.M. Chen, “Ontology-based concept map for planning a personalised learning path,” British Journal of Educational Technology, Vol. 40, no. 6, pp. 1028-1058, 2009. Article (CrossRef Link).

[6] M. Pushpa., “ACO in e-Learning: Towards an adaptive learning path,” International Journal on Computer Science \& Engineering, Vol. 4, no. 3, pp. 458-462, 2012.

[7] E. Kurilovas, I. Zilinskiene and V. Dagiene, "Recommending suitable learning scenarios according to learners' preferences: An improved swarm based approach,” Computers in Human Behavior, Vol. 30, pp. 550-557, 2014. Article (CrossRef Link).

[8] T.C. Hsieh, M.C. Lee and C.Y. Su, "Designing and implementing a personalized remedial learning system for enhancing the programming learning,” Educational Technology \& Society, Vol. 16, no. 4, pp.32-46, 2013. Article (CrossRef Link).

[9] P. Karampiperis and D. Sampson, "Adaptive Learning Re-sources Sequencing in Educational Hypermedia Systems,” Educational Technology \& Society, Vol. 8, no. 4, pp.128-147, 2005. Article (CrossRef Link).

[10] C.M. Chen, "Intelligent web-based learning system with personalized learning path guidance," Computers \& Education, Vol. 51, no. 2, pp. 787-814, 2008. Article (CrossRef Link).

[11] T.C. Yang, G.J. Hwang and J.H. Yang, "Development of an Adaptive Learning System with Multiple Perspectives based on Students' Learning Styles and Cognitive Styles,” Educational Technology \& Society, Vol. 16, no. 4, pp. 185-200, 2013. Article (CrossRef Link).

[12] G. Durand, N. Belacel and F. Laplante, "Graph theory based model for learning path recommendation,” Journal of Information Science, Vol. 251, no. 4, pp. 10-21, 2013. Article (CrossRef Link).

[13] D. Churchill, “Towards a Useful Classification of Learning Objects,” Educational Technology Research and Development, Vol. 55, no. 5, pp. 479-497, 2007. Article (CrossRef Link).

[14] S. Al-Muhaideb and M.E.B. Menai, "Evolutionary computation approaches to the Curriculum Sequencing problem,” Natural Computing, Vol. 10, no. 2, pp. 891-920, 2011. Article (CrossRef Link).

[15] Klašnja-Milićević, B. Vesin, M. Ivanović and Z. Budimac, "E-Learning personalization based on hybrid recommendation strategy and learning style identification," Computers \& Education, Vol. 56, no. 3, pp. 885-899, 2011. Article (CrossRef Link).

[16] Y.S. Lin, Y.C. Chang and C.P. Chu, "An Innovative Approach to Scheme Learning Map Considering Tradeoff Multiple Objectives,” Educational Technology \& Society, Vol. 19, no. 1, pp. 142-157, 2016. Article (CrossRef Link).

[17] C.F. Lin, Y.C. Yeh, Y.H. Hung and R.I. Chang, "Data mining for providing a personalized learning path in creativity: An application of decision trees,” Computers \& Education, Vol. 68, no. 4, pp. 199-210, 2013. Article (CrossRef Link).

[18] N.V. Jungum, N. Mohamudally and N. Nissanke, "Partitioning Application Using Graph Theory for Mobile Devices in Pervasive Computing Environments,” Procedia Computer Science, Vol. 94, pp. 105-112, 2016. Article (CrossRef Link). 
[19] D. Mears and H.B. Pollard, "Network science and the human brain: Using graph theory to understand the brain and one of its hubs, the amygdala, in health and disease," Journal of Neuroscience Research, Vol. 94, no. 6, pp. 590-605, 2016. Article (CrossRef Link).

[20] M. Alian and R. Jabri, "A shortest adaptive learning path in eLearning systems: mathematical view,” Journal of American Science, Vol. 5, no. 6, pp. 32-42, 2009.

[21] I.A. Alshalabi, S. Hamada and K. Elleithy, "Automated adaptive learning using smart shortest path algorithm for course units,” in Proc. of IEEE Conf. on Systems, Applications and Technology, pp. 1-5, 2015. Article (CrossRef Link).

[22] J.D. Novak and A.J. Ca-as, "The origins of the concept mapping tool and the continuing evolution of the tool,” Information Visualization, Vol. 5, no. 3, pp. 175-184, 2006. Article (CrossRef Link).

[23] D.A. Wiley, "Connecting learning objects to instructional design theory: A definition, a metaphor, and a taxonomy,” D.a.wiley the Instructional Use of Learning Objects, 2000.

[24] E. Kurilovas, I. Zilinskiene and V. Dagiene, "Recommending suitable learning paths according to learners’ preferences: Experimental research results,” Computers in Human Behavior, Vol. 51, pp. 945-951, 2015. Article (CrossRef Link).

[25] S.S. Nash, "Learning objects, learning object repositories, and learning theory: Preliminary best practices for online courses,” Interdisciplinary Journal of Knowledge \& Learning Objects, Vol. 1, no. 1, pp. 217-228, 2005. Article (CrossRef Link).

[26] Q. Lin, J. Chen, Z.H. Zhan, W.N. Chen, C.A.C. Coello, Y. Yin, C.M. Lin and J. Zhang, “A Hybrid Evolutionary Immune Algorithm for Multi-objective Optimization Problems,” IEEE Transactions on Evolutionary Computation, Vol. 20, no. 5, pp. 711-729, 2015. Article (CrossRef Link).

[27] J.T. Tsai, W.H. Ho, T.K. Liu and J.H. Chou, "Improved immune algorithm for global numerical optimization and job-shop scheduling problems,” Applied Mathematics \& Computation, Vol. 194, no. 2, pp. 406-424, 2007. Article (CrossRef Link).

[28] Q. Lin, Q. Zhu, P. Huang, J. Chen, Z. Ming and J. Yu, “A novel hybrid multi-objective immune algorithm with adaptive differential evolution,” Computers \& Operations Research, Vol. 62, no. C, pp. 95-111, 2015. Article (CrossRef Link).

[29] G. Pan, K. Li, A. Ouyang and K. Li, "Hybrid immune algorithm based on greedy algorithm and delete-cross operator for solving TSP,” Soft Computing, Vol. 20, no. 2, pp. 555-566, 2016. Article (CrossRef Link).

[30] C.F. Lin, Y.C. Yeh, Y.H. Hung and R.I. Chang, "Data mining for providing a personalized learning path in creativity: An application of decision trees," Computers \& Education, Vol. 68, no. 4, pp. 199-210, 2013. Article (CrossRef Link).

[31] Y. Akbulut and C.S. Cardak, “Adaptive educational hyper-media accommodating learning styles: A content analysis of publications from 2000 to 2011,” Computers \& Education, Vol. 58, no. 2, pp. 835-842, 2012. Article (CrossRef Link).

[32] R.M. Felder and B.A. Soloman, "Learning styles and strate-gies,” 2000. Article (CrossRef Link).

[33] U. Ocepek, Bosni, Zoran, Nan, Ovska, I. Erbec and J. Rugelj, "Exploring the relation between learning style models and preferred multimedia types,” Computers \& Education, Vol. 69, no. 4, pp 343, 2013. Article (CrossRef Link).

[34] Y.C. Lin, Y.T. Lin and Y.M. Huang, "Development of a diagnostic system using a testing-based approach for strengthening student prior knowledge,” Computers \& Education, Vol. 57, no. 2, pp. 1557-1570, 2011. Article (CrossRef Link).

[35] S.H. Liu and G.G. Lee, "Using a concept map knowledge management system to enhance the learning of biology,” Computers \& Education, Vol. 68, no. 4, pp. 105-116, 2013. Article (CrossRef Link).

[36] S. Schaal, F.X. Bogner and R. Girwidz, "Concept Mapping Assessment of Media Assisted Learning in Interdisciplinary Science Education,” Research in Science Education, Vol. 40, no. 3, pp. 339-352, 2010. Article (CrossRef Link).

[37] M. Neshat, G. Sepidnam, M. Sargolzaei and A.N. Toosi, "Artificial fish swarm algorithm: a survey of the state-of-the-art, hybridization, combinatorial and indicative applications," Artificial Intelligence Review, Vol. 42, no. 4, pp. 965-997, 2014. Article (CrossRef Link). 
[38] S.A. Hofmeyr and S. Forrest, "Computer immunology," Communications of the ACM, Vol 40, pp. 88-96, 1997. Article (CrossRef Link).

[39] S. Aine, R. Kumar and P.P. Chakrabarti, “Adaptive parameter control of evolutionary algorithms to improve quality-time trade-off,” Applied Soft Computing Journal, Vol. 9, no. 2, pp. 527-540, 2009. Article (CrossRef Link).

[40] K.A.D. Jong, “Analysis of the behavior of a class of genetic adaptive systems," Dept Computer And communication Sciences University of Michigan, 1975. Article (CrossRef Link).

[41] R.M. Gagne, L.J. Briggs, "Principles of instructional design,” Performance Improvement, Vol. 44, no. 2, pp. 44-46, 1974. Article (CrossRef Link).

[42] K. Hilal, A. Karamete and O. Aydin, "The Effects of a Computer-Assisted Teaching Material, Designed According to the ASSURE Instructional Design and the ARCS Model of Motivation, on Students' Achievement Levels in a Mathematics Lesson and Their Resulting Attitudes,” European Journal of Contemporary Education, Vol. 15, no. 1, pp. 105-113, 2016. Article (CrossRef Link).

[43] Django Project, 2018. Article (CrossRef Link).
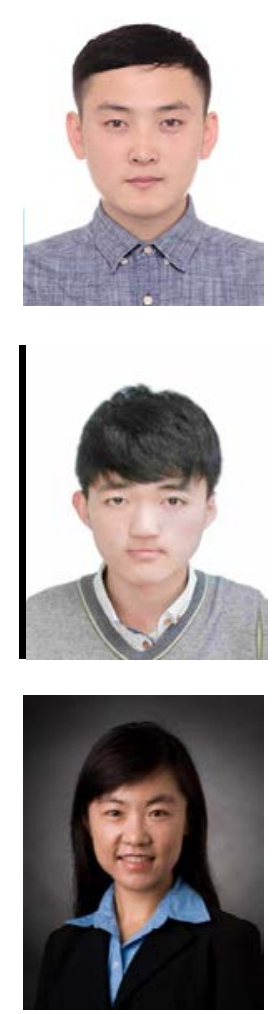

Cun-Ling BIAN, received his B.S. degree in Educational Technology from Ocean University of China, China, in 2016. He is currently a Master candidate in Educational Technology, in Ocean University of China. His research interests include machine learning,computer vision and adaptive learning.

De-Liang WANG, a senior student, is currently a Bachelor candidate in Educational Technology, in Ocean University of China. His research interests include machine learning and adaptive learning.

Shi-Yu LIU, received her Ph.D. degree in Educational Psychology from the University of Minnesota, U.S., in 2014. She is currently an assistant professor at Ocean University of China. Her research interests include student learning, educational assessment, and curriculum development. 


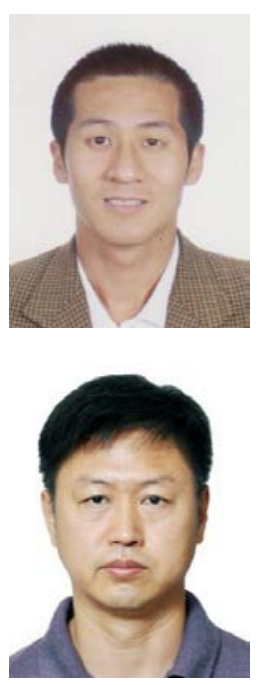

Wei-Gang LU, received his Ph.D.degree in Computer Application Technology from Harbin Institute of Technology, China, in 2012. He is currently an Associate Professor in Ocean University of China. His research interests include learning system modeling, scientific data visualization, and machine learning.

Jun-Yu DONG, received his Ph.D. degree in Image Processing from Heriot-Watt University, UK, in 2003. He is currently a Professor and the head of the Department of Computer Science and Technology in Ocean University of China. His research interests include machine learning. 